\title{
Prospects for CP violation measurements with charmless hadronic B meson decays
}

\section{Ignacio Bediaga on behalf of LHCb collaboration*}

Centro Brasileiro de Pesquisas Fisicas

bediaga@cbpf.br

Studies of two and three-body charmless hadronic decays of B mesons have high sensitivity to possible contributions from New Physics. Precision measurements involving these decays will be performed at $\mathrm{LHCb}$, notably those of CP-violating asymmetries. These measurements benefit from a trigger system which is very efficient for hadronic final states and excellent particle identification capabilities. The full programme is reviewed and first studies with 2010 data are presented.

35th International Conference of High Energy Physics - ICHEP2010,

July 22-28, 2010

Paris France

* Speaker. 


\section{Introduction}

Charmless B non-leptonic decays offer many opportunities to search for effects of CP violation which may or may not be describable by the Standard Model CKM paradigm. Tree and penguin processes contribute simultaneously in many of these decays, allowing interference between them and consequently the possibility arises for $\mathrm{CP}$ violation observables to manifest themselves. In these decays the CKM weak phase $\gamma$ is present through the tree contribution. On the other hand the penguin contribution involves loops that allows possibles contributions from new physics. Due the low branching fraction of these channels, high statistics, good detector and hadronic trigger performance are required to reveal evidence of possible new sources of $\mathrm{CP}$ violation. These requirements are satisfied at the LHCb experiment [1]. In this report we review the physics programme of $\mathrm{LHCb}$ in this sector and show results obtained with early data.

The initial strategy of LHCb in this class of decay is to concentrate on two and three body modes involving kaons, pions and protons in the final state. With $\sim 1 f b^{-1}$, which is the integrated luminosity expected in 2011, we expect to collect one order of magnitude more events for these decays than have been observed at previous experiments. It will also be possible to improve the sensitivity in searches for suppressed decays such $B^{0} \rightarrow p \bar{p}, B_{s}^{0} \rightarrow \pi^{+} \pi^{-}$and $B^{0} \rightarrow K^{+} K^{-}$, and highly suppressed decays such $B^{+} \rightarrow \pi^{-} K^{+} K^{+}$[3], whose branching ratio may be enhanced in certain new physics scenarios.

\section{Analysis plans}

The simplest and straightforward analysis for two body decays involves the untagged and time independent measurement of the CP asymmetries $\mathscr{A}_{C P}\left(B_{s}^{0} \rightarrow \pi^{+} K^{-}\right), \mathscr{A}_{C P}\left(\Lambda_{b}^{0} \rightarrow p \pi^{-}\right), \mathscr{A}_{C P}\left(\Lambda_{b}^{0} \rightarrow\right.$ $\left.p K^{-}\right)$and $\mathscr{A}_{C P}\left(B^{0} \rightarrow K^{+} \pi^{-}\right)$, the latter of which is involved in the well known ' $\mathscr{A}_{C P}$ puzzle' [4].

For $B^{+}$decays into three charged hadrons, we are considering the decays: $B^{ \pm} \rightarrow \pi^{ \pm} \pi^{\mp} \pi^{ \pm}$, $B^{ \pm} \rightarrow K^{ \pm} \pi^{\mp} \pi^{ \pm}, B^{ \pm} \rightarrow K^{ \pm} K^{\mp} \pi^{ \pm}, B^{ \pm} \rightarrow K^{ \pm} K^{\mp} K^{ \pm}, B^{ \pm} \rightarrow \pi^{ \pm} p \bar{p}$ and $B^{ \pm} \rightarrow \pi^{ \pm} p \bar{p}$. We will use the Mirandizing method [5] to search for possible sources of CP violation coming from interference between two neighbouring resonances or even from different amplitudes of a single intermediate resonant state.

An interesting study that will be performed with early data is the measurement of the $B_{s}^{0}$ lifetime using the decay mode $B_{s}^{0} \rightarrow K^{+} K^{-}$. This observable is directly related to the value of $\Delta \Gamma_{s}$ in the limit where the tree contribution is negligible. To facilitate this measurement a special trigger is being implemented to avoid bias at low $B_{s}^{0}$ lifetimes.

The collaboration is also preparing for more complex analyses involving tagged and time dependent $\mathrm{CP}$ asymmetry studies for $B_{s}^{0}$ and $B^{0}$ two body decays, which will enable the determination of the CKM $\gamma$ phase through U-spin symmetry [6].

In the field of three-body decays we are developing a Dalitz analysis to measure the $\mathrm{CP}$ asymmetry in resonant intermediate states $\mathscr{A}_{C P}\left(B^{+} \rightarrow\right.$ Resonance $\left.{ }^{0} h^{+}\right)$(here 'resonance' indicates a low mass two-body scalar, vector or tensor intermediate state) and also to extract the $\gamma$ phase associated with the $B^{0} \rightarrow K_{s}^{0} \pi^{\mp} \pi^{ \pm}$decay [7]. 


\section{Selection strategy}

Inclusive preselection strategies are used for both the two and three body decays studies. These are based on a two or three charged tracks selection without particle identification, assigning all tracks the pion mass hypothesis. These inclusive selections are based only on vertex cuts. (The only exception to this general approach is in the selection of $B_{s}^{0} \rightarrow K^{+} K^{-}$decays for lifetime measurements, where RICH information is indeed used.) The discrimination variables used in this preselection include transverse momentum, impact parameter, displacement significance and vertex $\chi^{2}$. The success of this approach is reliant on the LHCb good track and mass resolution [8].

The final selection makes use of the excellent particle identification provided by the RICH system [9]. The clear separation between pions, kaons and protons allows a very cleans invariant mass signal to be obtained for each decay with little loss of efficiency.

\section{First data and expected yields}

The LHC run which began this year is expected to continue until the end of 2011. The expected accumulated luminosity is approximately $1 \mathrm{fb}^{-1}$. Taking in account the preliminary LHCb measurement of the b-production cross section at $\sqrt{s}=7 \mathrm{TeV}$ [10], we can expect to have at the end of this run, about a hundred thousand reconstructed events for the higher branching fractions $\left(\sim 10^{-5}\right)$ charmless B-decay channels.

Already the first preliminary charmless B decay signal has been found by LHCb. Figure 1 shows the invariant mass spectrum for $B^{0} \rightarrow K^{+} \pi^{-}$selection with an accumulated luminosity of $122 \mathrm{nb}^{-1}$. A total $15.1 \pm 4.3$ events are observed, consistent with expectations.

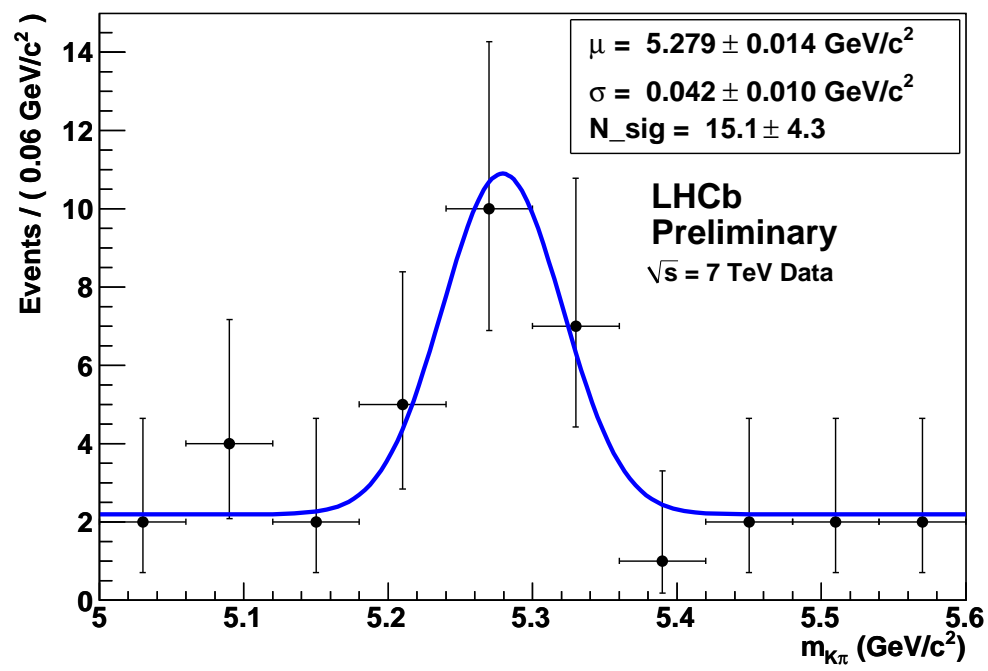

Figure 1: First charmless $B^{0} \rightarrow K^{+} \pi^{-}$decay signal, with an accumulated luminosity of $122 \mathrm{nb}^{-1}$ 


\section{Expected CP reach in two-body modes}

With the anticipated number of events in the two-body charmless decays, there will be with $1 \mathrm{fb}^{-1}$ of data a substantial improvement in the precision of many CP asymmetries, in particular those involving $\Lambda_{b}^{0}$ and $B_{s}^{0}$. Assuming this integrated luminosity, and assuming systematics errors to be small, one can anticipate the following reduction in uncertainty from the existing value[2]:

- $\mathscr{A}_{C P}\left(B^{0} \rightarrow K^{+} \pi^{-}\right)$from $-0.0980 \pm 0.012$ to \pm 0.004

- $\mathscr{A}_{C P}\left(B_{s}^{0} \rightarrow \pi^{+} K^{-}\right)$from $0.39 \pm 0.15$ to \pm 0.025

- $\mathscr{A}_{C P}\left(\Lambda_{b}^{0} \rightarrow p \pi^{-}\right)$from $0.03 \pm 0.17$ to \pm 0.025

- $\mathscr{A}_{C P}\left(\Lambda_{b}^{0} \rightarrow p K^{-}\right)$from $0.37 \pm 0.17$ to \pm 0.015

For the observation of the decay $B^{0} \rightarrow p \bar{p}$ with a signal significance of five standard deviation, LHCb simulation studies suggest that discovery will be possible in 2011 if this decay has a branching fraction bigger than $410^{-8}$. The present upper limit obtained by Belle is $1.110^{-7}$ [11].

\section{Search for CP violation sources in Dalitz three body $B^{+}$decay.}

The resonance structures present in three body charmless $B^{+}$decays can be a rich field for $\mathrm{CP}$ studies. The intermediate state can be produced through different amplitudes with possible different weak phase contributions. Inside the Dalitz plot the shape of the interference between two intermediate states that occupy the same phase space region depends directly from the phase difference. Weak phases change sign depending on whether the parent meson is a $B^{+}$or $B^{-}$, and so the interference between diagrams involving a weak phase and those that do not will lead to a net amplitude that is different for the two cases. Therefore, the signature of $\mathrm{CP}$ violation in three body decays is a difference between the $B^{+}$and $B^{-}$Dalitz surfaces [5].

Amplitude analysis have been used to extract information from three body $\mathrm{B}$ decays in order to identify possible sources of $\mathrm{CP}$ violation. However such an analysis is typically a long term project, which often involves non-trivial theoretical problems in defining and interpreting the model used to perform the amplitude fit.

For this reason it is decided that in the first analysis of $\operatorname{LHCb} B \rightarrow$ hhh data, a model independent approach will be employed to search for statistically significant Dalitz differences between the charged conjugate three body decays. The Mirandizing approach [5] takes each Dalitz surface, divides it into $i$ bins, $N(i)$ for $B^{+}$Dalitz and $\bar{N}(i)$ for $B^{-}$and computes the CP bin significance distribution ${ }^{D P} S_{C P}(i)$.

$$
{ }^{D P} S_{C P}(i) \equiv \frac{N(i)-\bar{N}(i)}{\sqrt{N(i)+\bar{N}(i)}}
$$

which gives the amount of standard deviation assuming a Poissonian distribution. The ${ }^{D P} S_{C P}$ distribution must be a Gaussian centred on zero and of width $1(\mathrm{~g}(0,1))$, for two statistically equivalent Dalitz surfaces, which would be the case for a CP conserving decays. Otherwise, in the presence of a CP violating source, the ${ }^{D P} S_{C P}$ distribution will be different from a $\mathrm{g}(0,1)$. With the large 
statistics samples we expect at $\mathrm{LHCb}$, we will be able to subdivide the Dalitz plot into different regions to identify what resonance or resonances are the origin of the $\mathrm{CP}$ violation.

For the 2011 run with an accumulated luminosity of $1 \mathrm{fb}^{-1}$, we can expect to study possible sources of CP violation for the decays $B^{ \pm} \rightarrow \pi^{ \pm} \pi^{\mp} \pi^{ \pm}, B^{ \pm} \rightarrow K^{ \pm} \pi^{\mp} \pi^{ \pm}$and $B^{ \pm} \rightarrow K^{ \pm} K^{\mp} K^{ \pm}$, all of which have branching fractions bigger than $10^{-5}$. For the decays $B^{ \pm} \rightarrow K^{ \pm} K^{\mp} \pi^{ \pm}, B^{ \pm} \rightarrow \pi^{ \pm} p \bar{p}$ and $B^{ \pm} \rightarrow \pi^{ \pm} p \bar{p}$ the sensitivity will be lower.

\section{References}

[1] A. Augusto Alves Jr et al. LHCb Collaboration, JINST 03 S08005 (2008).

[2] K. Nakamura et al. Particle Data Group, Journal of Physics G, 37075021 (2010).

[3] B. Aubert et al. BABAR Collaboration, Phys. Rev.D78, 091102(R) (2008).

[4] S.-W. Lin et al. Belle Collaboration, Nature 452, 332 (2008).

[5] I. Bediaga, I.I. Bigi, A. Gomes, G. Guerrer, J. Miranda and A.C.dos Reis, Phys.Rev.D80 096006,(2009).

[6] R. Fleischer, Phys. Lett. 459B, 306 (1999).

[7] I. Bediaga, G. Guerrer, J. Miranda, Phys.Rev.D76, 073011, (2007).

[8] S. Boghi, on behalf of LHCb Collaboration, this proceedings.

[9] A. Powell, on behalf of LHCb Collaboration, this proceedings.

[10] S. Stone, on behalf of LHCb Collaboration, this proceedings.

[11] Y.-T. Tsai, P. Chang, et al. Belle Collaboration, Phys. Rev. D75, 111101 (2007). 